\title{
Minding the gap: in-flight body awareness in birds
}

Ingo Schiffner ${ }^{1 *}$, Hong D Vo ${ }^{1}$, Partha S Bhagavatula ${ }^{1}$ and Mandyam V Srinivasan ${ }^{1,2,3}$

\begin{abstract}
Introduction: When birds fly in cluttered environments, they must tailor their flight to the gaps that they traverse. We trained budgerigars, Melopsittacus undulatus, to fly through a vertically oriented gap of variable width, to investigate their ability to perform evasive manoeuvres during passage.

Results: When the gap was wider than their wingspan, the birds passed through it without interrupting their flight. When traversing narrower gaps, however, the birds interrupted their normal flight by raising their wings or tucking them against the body, to prevent contact with the flanking panels. Our results suggest that the birds are capable of estimating the width of the gap in relation to their wingspan with high precision: a mere $6 \%$ reduction in gap width causes a complete transition from normal flight to interrupted flight. Furthermore, birds with shorter wingspans display this transition at narrower gap widths.

Conclusion: We conclude from our experiments that the birds are highly aware of their individual body size and use precise, anticipatory, visually based judgements to control their flight in complex environments.
\end{abstract}

Keywords: Birds, Flight, Vision, Body awareness, Obstacle avoidance

\section{Introduction}

When traversing cluttered environments at nearly cruising speeds, birds need to be constantly aware of the distances to oncoming obstacles and the spaces between them, in order to make split second decisions about whether a gap can be traversed, and to determine whether a change in the wing posture is necessary to facilitate an injury-free passage (e.g. [1]).

Do birds fly through passages that are narrower than their wingspan? If they indeed do so, what postural changes do they make to accommodate the passage? Are the wings held up, held down, held forward, or held behind, tucked close to the body? Furthermore, very little is known about a bird's ability to assess the width of a gap in relation to its own body size, and about how this assessment is made. In principle, there are a number of ways in which this could be accomplished. For example, the width of the gap could be judged from afar, by using vision to gauge the angular width of the gap and the viewing distance - from which the absolute width of the

\footnotetext{
* Correspondence: i.schiffner@uq.edu.au

${ }^{1}$ Queensland Brain Institute, University of Queensland, St Lucia QLD 4072, Australia

Full list of author information is available at the end of the article
}

gap can be estimated. Alternatively - as explained in the Discussion - this judgement could be made by measuring the rate at which the visual image of the gap expands when it subtends a prescribed visual angle. A third possibility is that the width of the gap is estimated just as it is being traversed, using vision to determine whether the wing tips are about to touch or clear the edges of the gap (For a general introduction to bird vision and its possible role in collision avoidance see [2,3]).

In the experiments presented here, seven budgerigars (Melopsittacus undulatus) were confronted with an aperture of variable width. We aimed to investigate their flight manoeuvres through the aperture and to enquire whether they display awareness of their body size while doing so. The aperture was a vertical slit, presented as a gap between two cloth panels (see Figure 1). A total of 560 flights were recorded, with 10 flights per bird in seven experimental and one control (unobstructed tunnel) condition.

\section{Results}

During the normal flapping flight mode - when the birds were not negotiating an aperture - the birds' wingbeat cycles proved to be very stable, with the duration of 


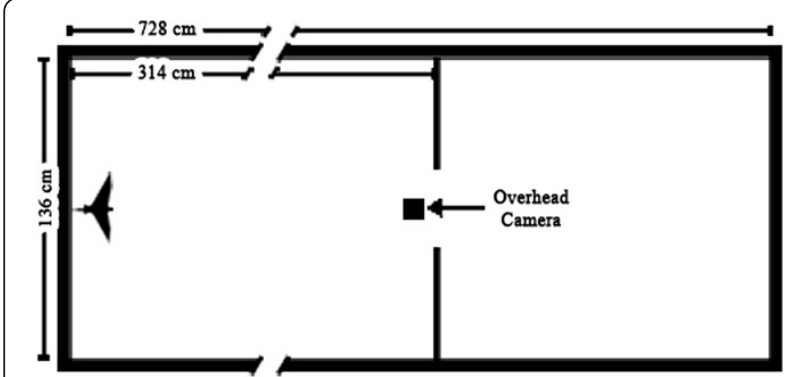

Figure 1 Setup of the experimental tunnel. Shown are the starting position of the bird, and the position of the gap and the camera (length not to scale).

the downstrokes being in the range of $38 \pm 3 \mathrm{~ms}$ and the upstrokes in the range of $22 \pm 2 \mathrm{~ms}$, resulting in a mean wingbeat period of approximately $60 \mathrm{~ms}$. Even though there was a slight variation in the duration of the downstroke and the upstroke, the ratio between the two remained almost constant at $0.59 \pm 0.07$. These figures were constant across individuals and across different experimental conditions.

As the width of the gap was reduced to approach the wingspan of each individual bird, the normal wingbeat cycle was interrupted during the actual passage through the gap. The duration of the upstroke was then longer than the duration of the downstroke, indicating that the birds actively held their wings in such position to avoid touching the panels during the passage. During traversal of the gap, the birds either held their wings in a position corresponding to the end of an upstroke, or tucked them in against the body (a behaviour very reminiscent of flap bounding, i.e. intermittent phases during which normal flapping flight is interrupted; see [4]). In either case, the birds closed their wings, 'projectiling' themselves through the gap, rather than actively flapping through it (see Figure 2 or the videos included as Additional files 1 and 2). The choice of the mode of traversal depended upon the duration of the passage: during longer traversals the wings were always tucked in completely. Only in one instance (out of a total of 490 narrow-gap traversals) did we observe a bird holding its wings pointing downwards, and once a bird holding one wing up and the other down. For simplicity, we shall refer to all of the 'projectiling' behaviours as 'wing closure'.

Mechanical contact with the cloth panel occurred in only $8 \%$ of the 490 gap traversals i.e. all trials excluding the flights in the unobstructed tunnel. No major collisions where observed in any of the flights. This result clearly indicated that budgerigars are capable of flying through passages that are narrower than their wingspan without injuring themselves.

We found that, as the gap was made narrower, the birds were more likely to interrupt their wingbeat cycle
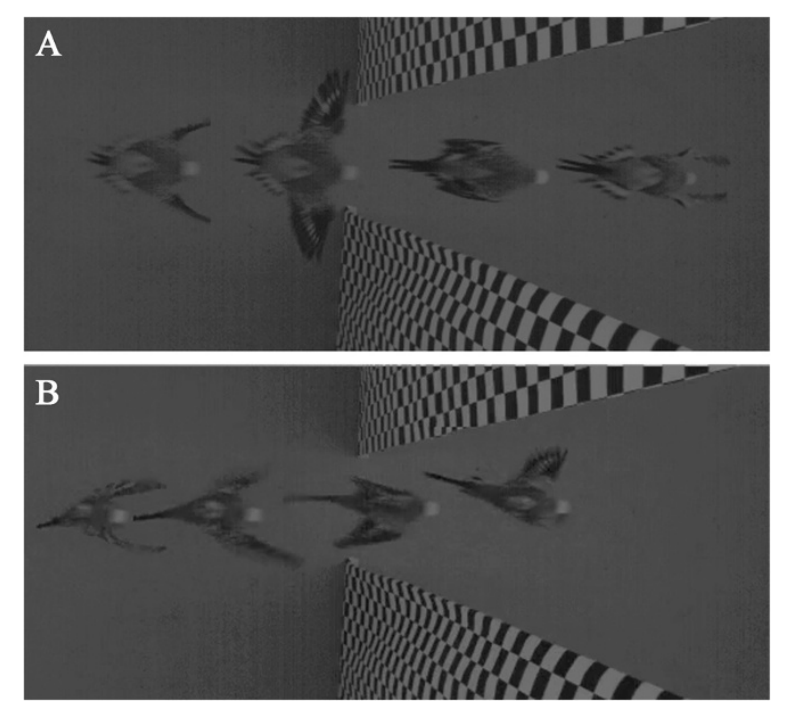

Figure 2 Examples of high-speed videography of the budgerigars' flights while traversing the gap. Panel (A) shows a bird 'projectiling' through the gap with its wings almost completely tucked back. Bird shown is Drongo (wingspan $31 \mathrm{~cm}$ ), the gap width in the example is $28 \mathrm{~cm}$. Panel (B) shows a budgerigar traversing the gap with its wings held above its body for a prolonged time. Bird shown is One (wingspan $31 \mathrm{~cm}$ ), the gap width is $28 \mathrm{~cm}$. The individual frames display the bird's posture at the end of each downstroke, before and after the gap and additionally the body-posture during traversal (full videos can be found in the Additional files 1 and 2).

and close their wings. The variation of the probability of wing closure [(No. of observed wing closures / 10) * 100] with gap width is shown in Figure 3A (note that the abscissa in this figure represents the relative gap width, i.e. the difference between the bird's wingspan and the width of the gap - a negative value means that the gap is narrower than the wingspan). Furthermore, the birds maintained wing closure for a longer duration as the gap was made narrower. This is illustrated in Figure 3B, which shows how the normalised duration of the upstroke varies with gap width at the moment of passage. More importantly, the birds are not simply adjusting the phase of their normal wingbeat cycle so as to ensure that wings are in the closed position when passing through the gap. They are definitely prolonging the period of wing closure during passage through the gap. This is evinced by the fact that the normalised duration of the upstroke is about $70 \%$ when passing through the narrowest gap, as compared to about $20 \%$ when passing through the widest gap (see Figure $3 \mathrm{~B}$ ).

Both the probability of wing closure (One Way ANOVA: $\mathrm{F}(6,42)=8.539 ; \mathrm{p}<0.001)$ and the normalised duration of the upstroke phase during passage vary strongly with gap width (One way ANOVA: F $(6,42)=5.002 \mathrm{p}<0.001$ ). Both measures increase significantly as the width of the gap is decreased from -1 to $-3 \mathrm{~cm}$ (Wilcoxon signed rank test: 


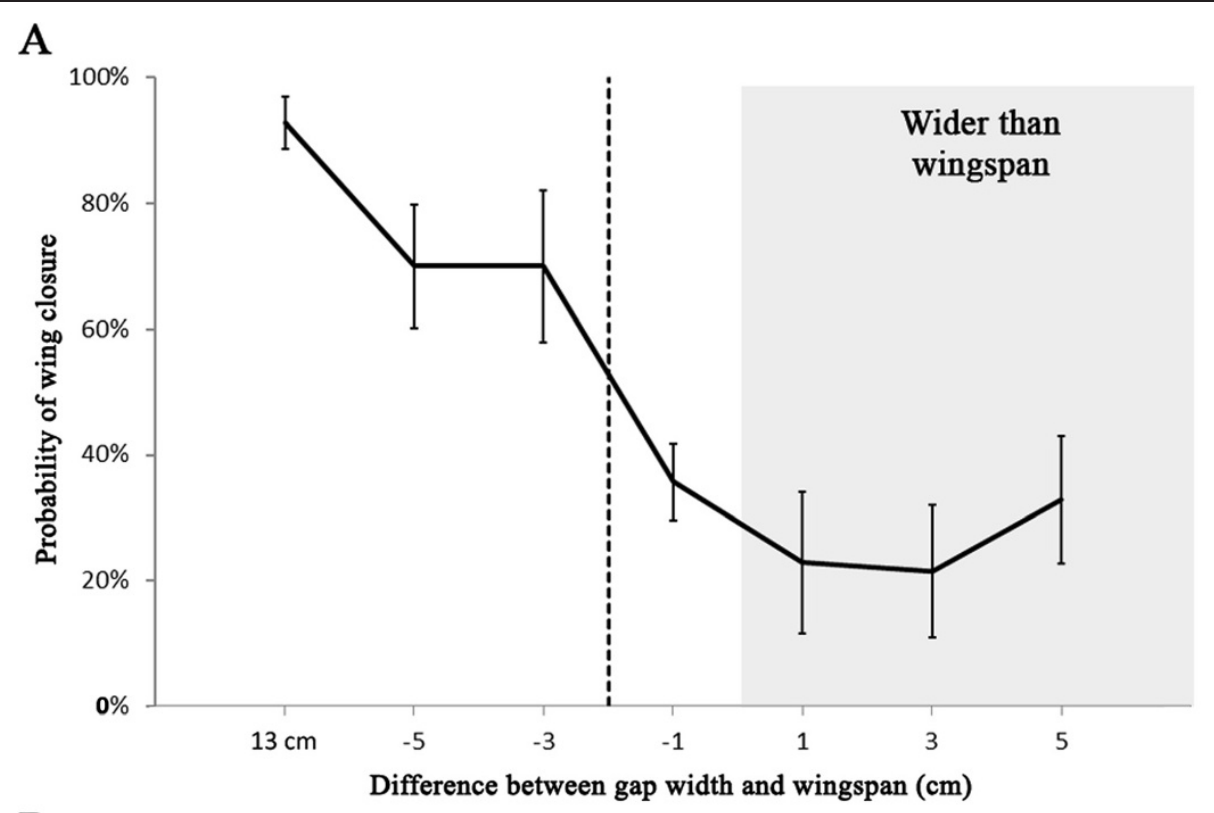

B

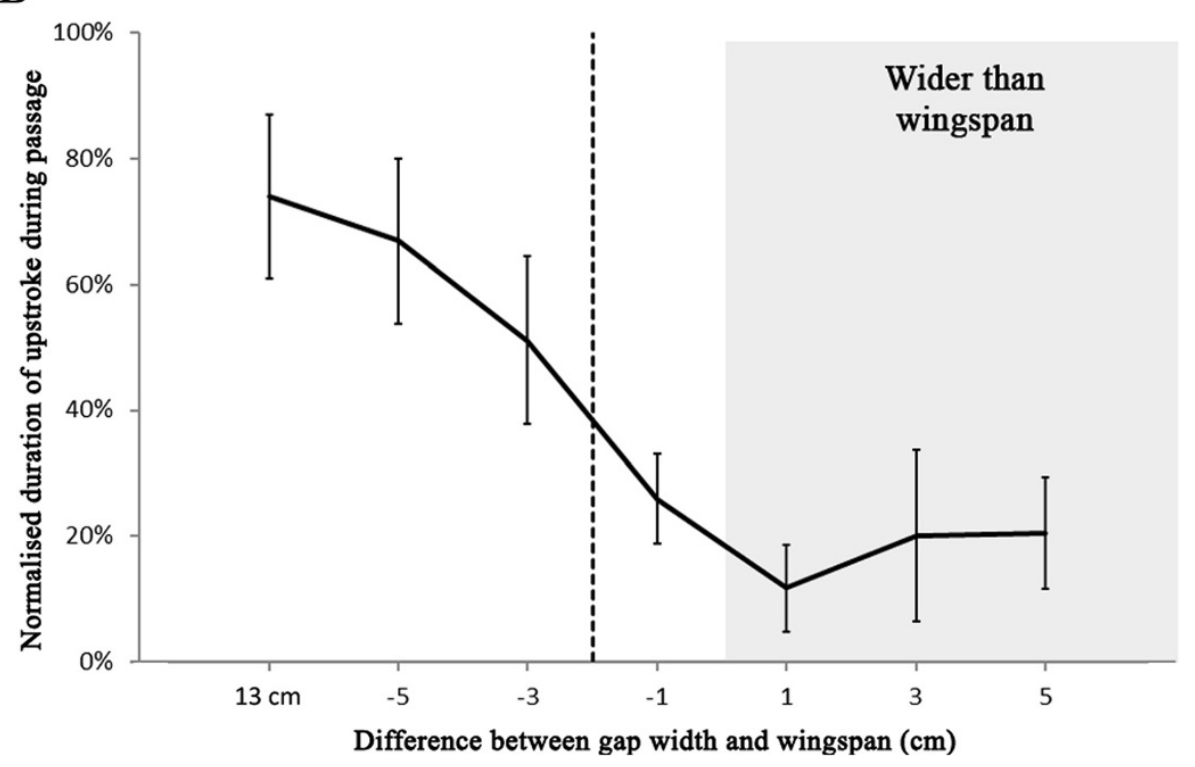

Figure 3 Mean probability of wing closure (A) and normalised mean duration of the up-stroke during passage (B) as a function of the difference between the gap width and the bird's wingspan, averaged over all 7 birds and including SEM. The percentage probability of wing closure for each bird was computed as $100^{*}$ (No. of observed wing closures/10), from the 10 flights that were analysed for each bird, for each condition. The grey area represents the conditions in which the gap was wider than the bird's wingspan.

$\mathrm{p}<0.05$ ), indicating a transition from uninterrupted to interrupted flight when the width of the gap is reduced to a value that is $2 \mathrm{~cm}$ narrower than the bird's wingspan, as shown by the dashed lines in Figure 3A and B. Thus, the birds are aware of their own wingspan with a precision of $+/-2 \mathrm{~cm}$. Inspection of the data with the birds divided into 3 groups according to their wingspans $(29,31$ and $33 \mathrm{~cm}$ ) as shown in Figure 4, suggests that the larger birds (33 $\mathrm{cm}$ wingspan) show a propensity to close their wings even when the gap is larger than their wingspan.
Furthermore, all three groups of birds exhibit a significant increase in the probability of wing closure when the gap becomes narrower than their respective wingspans (Figure 4).

In addition, we examined the probabilities of wing closure for the three groups of birds at each of the three critical gap widths $(28,30$ and $32 \mathrm{~cm})$, as shown in Figure 5. For any given gap width, larger birds are more likely to close their wings than smaller birds (Two way ANOVA: $F(2,12)=4.742 ; \mathrm{p}=0.0304)$, and close their wings for longer times (Two way ANOVA: $F(2,12)=$ 


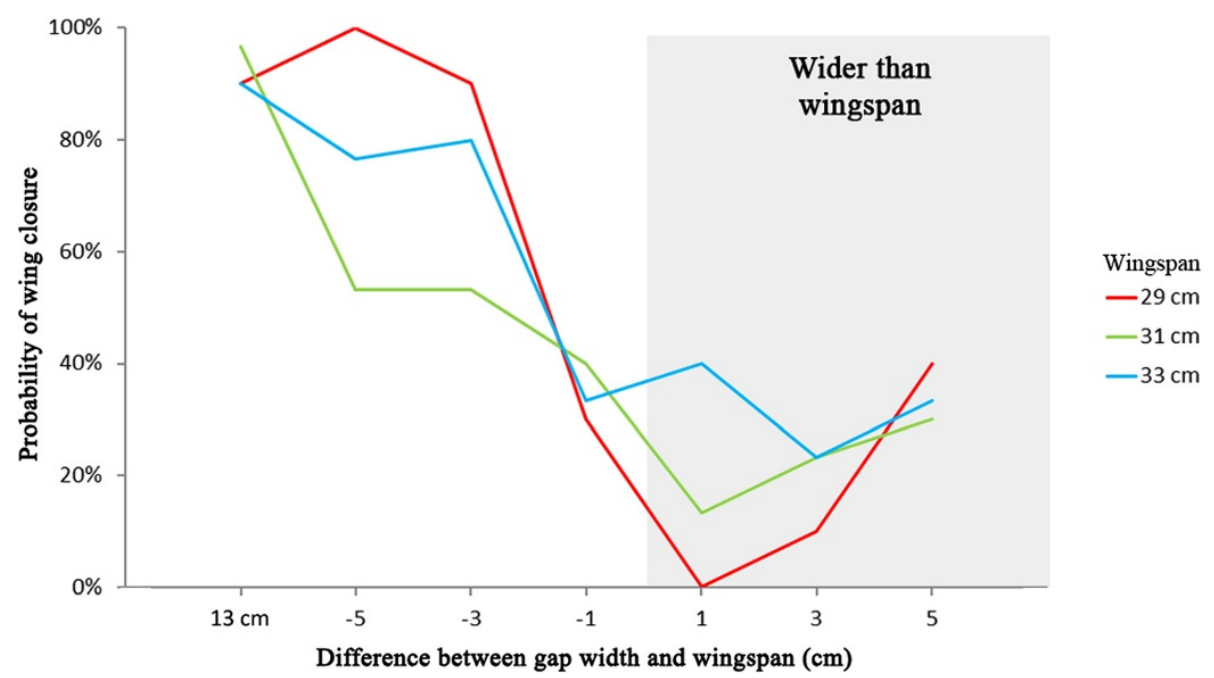

Figure 4 Probability of wing closure for birds with different wingspans. The graph shows the mean probability of wing closure for birds with wingspans of 29,31 and $33 \mathrm{~cm}$. Details are as in Figure 3.

8.722; $\mathrm{p}=0.00458)$. Thus, it would appear that each bird possesses a body template that is specific to its own wingspan: it does not simply close its wings when the width of the gap is lower than a certain absolute value.

When a gap induces wing closure, how far back along the approach trajectory does the wing closure commence? This can be estimated as the distance of the bird from the gap when the wings were fully outstretched for the last time before passing through the gap. Figure 6 shows a histogram of the distribution of these distances,

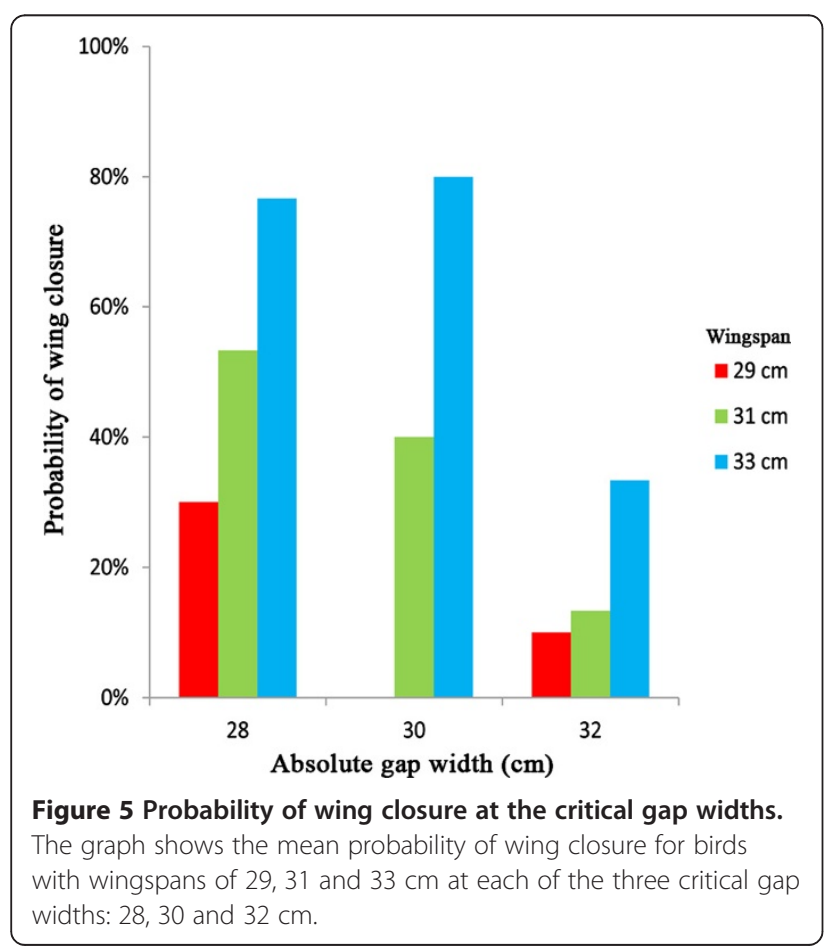

obtained by pooling data from all flights in which the birds closed their wings - as indicated by a longer upstroke duration-assuming a constant flight speed of $4 \mathrm{~m} / \mathrm{s}$. This histogram shows that, for these flights, the final wing extension occurred most frequently at a distance of about $220 \mathrm{~mm}$ from the gap. Furthermore, the cumulative histogram in Figure 6 shows that only 20\% of the final wing extensions occur at distances that are closer than $120 \mathrm{~mm}$ from the gap; $80 \%$ of these extensions occur at greater distances.

For comparison, Figure 7 shows the histogram of the distances of final wing extensions for gaps that did not induce wing closure. Here the histogram of final wingextension distances is approximately uniformly distributed over the entire period of an average, uninterrupted wing beat cycle. This wing-stroke period is approximately $60 \mathrm{~ms}$, as mentioned above, and is indicated by the vertical arrow on the time scale. The fact that the timing of the final wing extension is distributed more or less uniformly over the duration of an entire wing beat cycle indicates that this extension occurs independently of the distance of the bird from the aperture, and that it is a part of the normal wing beat cycle of the bird during uninterrupted flight. This is in contrast with the data in Figure 6, which show a non-uniform distribution of wing extensions over the period of a wing beat cycle, indicating that the narrower gaps are clearly affecting the wing beat cycle during the approach to the gap.

\section{Discussion}

The observation that birds close their wings when negotiating narrow gaps is not, in itself, surprising. This phenomenon would be apparent to anyone who watches a bird weave its way through dense foliage, and it is 

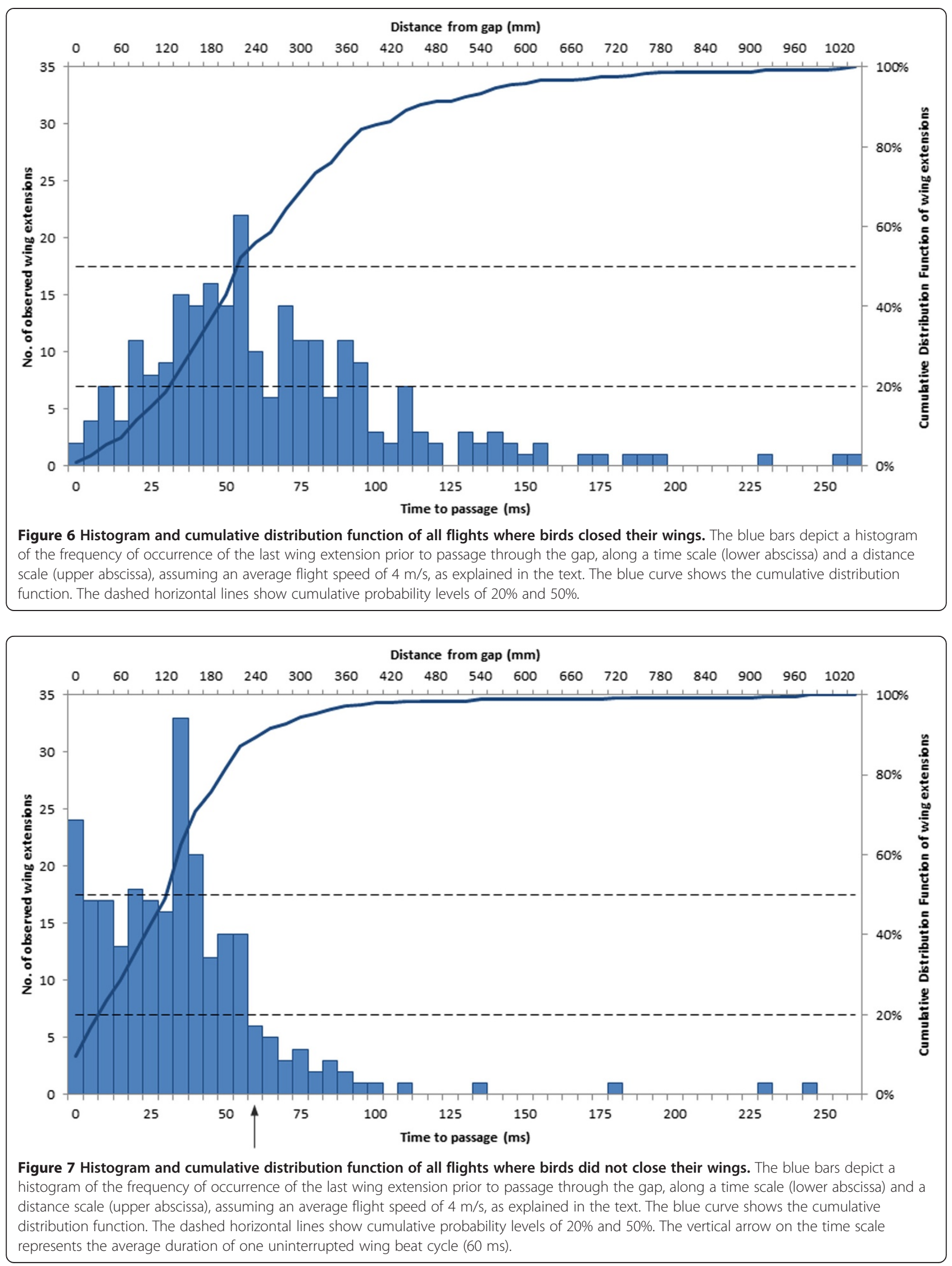
obviously a sensible thing to do to avoid injury. What is surprising, however, is the precise tuning of this behaviour to the width of the gap. Our results reveal that wing closure occurs only when the width of the gap approaches a value that is within $6 \%$ of the bird's wingspan. This means that the wings are never folded back unless it is absolutely necessary. Why don't birds adopt a safer, more conservative approach by closing their wings at larger gap widths? The answer may be that wing closure causes the bird to lose (a) altitude and (b) manoeuvrability. We have observed that the birds in fact lose altitude when they are projectiling [5]. Furthermore, we have also observed that when the birds approach a narrow gap they often increase their altitude prior to entering the gap, presumably compensating for the loss of altitude that is incurred during the passage in advance [5]. Thus, it may be important to ensure that the wings are closed only when absolutely necessary; and that the closure period is as brief as possible. In order to achieve this, birds need to be accurately aware of the relationship between their wingspan and the width of the gap.

Our study cannot answer the question as to whether birds are 'consciously' aware of their wingspans. Nevertheless, we have demonstrated that birds are able to tailor their behaviour in an accurate way to facilitate safe passage through narrow gaps. This must mean that precise information about body size is incorporated into their behaviour.

Awareness of body size has been studied mainly in humans (e.g. when walking through a narrow or short doorway, humans rotate their shoulders sideways if the doorway is too narrow, or duck if it is not high enough) and they appear to be able to predict in advance whether such accommodative movements are necessary [6-9]. Humans are also able to estimate, with an accuracy of about a centimetre, whether a hand will pass through an aperture [10]. Toads decide to either pass through a gap or to detour around it depending upon whether the gap is wider or narrower than $3 \mathrm{~cm}$, which is approximately the width of the head [11]. To the best of our knowledge, body size awareness has not been investigated in any other animal. Most studies in this general area have focused on the sensory aspects of obstacle detection, but not on how this information is linked to body size to facilitate collision-free navigation.

Our experiments reveal that birds are capable of estimating their own body size (wingspan) with a precision of $+/-2 \mathrm{~cm}$. Furthermore, birds are individually aware of their body size, with larger birds commencing wing closure at larger gap widths. It has been observed that humans perform accommodative shoulder rotations when preparing to walk through doorways that are narrower than $130 \%$ of their shoulder width, which allows for a considerable margin of error [6]. Our birds, on the other hand, begin to close their wings only when the gap is $2 \mathrm{~cm}$ (or ca. 6\%) narrower than the wingspan. The reasons for this less cautious behaviour in birds may be (i) a lower chance of serious injury or blockage of passage, as the wings are compliant structures; and (ii) with a free-running wingbeat cycle, the chances that the wings are fully extended exactly at the time of passage through the gap are rather low - the time-averaged distance between the wingtips would be approximately half the maximum wingspan, although the exact figure would depend upon the details of the wing kinematics.

In our experiments, the only information available to the birds to estimate the width of the gap are the visual cues carried by the checkerboard patterns on the panels that flank the gap. Boundary airflow effects near the edges of the gap are unlikely to be the triggers of wing closure, as the closure usually occurs well before the gap is traversed. Furthermore, collisions with the flanking panels occur substantially more frequently when they are devoid of visual texture (white). For flights through a $17 \mathrm{~cm}$ gap, the collision rate was $0 \%$ when both panels carried the checkerboard, and 23\% when both panels were white (data from 4 birds and a total of 70 flights; results taken from [5]).

What could be the nature of these visual cues? For humans walking through doorways, it has been suggested that intrinsic knowledge of the height of the eye above the ground, coupled with measurement of the visual angle subtended by the gap at any particular distance from the doorway, and the assumption of walking on a horizontal plane, should provide enough trigonometrical information to derive the distance to the doorway, and hence the width of the gap - see for example, [8]. However, it is unlikely that birds use the same strategy because their flights are of variable height and the ground cannot always be approximated by a horizontal plane. Alternatively, birds may use stereo information to determine the distance to the gap, and to combine this with information on the angular subtense of the gap to estimate the physical width of the gap. While this is theoretically possible, good stereo vision is unlikely to be present in budgerigars, given the low acuity and small region of binocular overlap in the frontal visual fields of most non-predatory birds [2].

Another possibility to be considered is that the birds are directly comparing the size of their wingspans with the width of the gap, just prior to traversing the gap. However, this is very unlikely because, during the passage through the gap, each wingtip and the corresponding gap edge would be in the lateral field of view, and would therefore be viewed by one eye only. This would preclude the acquisition of stereo cues. With monocular vision there would be no distance cues, consequently it would be impossible to gauge whether a wingtip extends beyond the edge of the gap, even when these two 
features are closely juxtaposed in the retinal image. Furthermore, as we have mentioned above, under the conditions in which the birds close their wings whilst flying through the gap, only $20 \%$ of the final wing extensions occur at distances that are closer than $120 \mathrm{~mm}$ from the gap; and $80 \%$ of these wing extensions occur at distances greater than $120 \mathrm{~mm}$ from the gap. These figures make it very improbable that the relationship between wingspan and gap width is gauged by a direct visual comparison, because in most of the instances, the bird is too far away from the gap to be able to make such a comparison.

It is possible that the birds are able to extract information about the width of the gap from the rate at which the image of the gap widens as the gap is approached. It can be shown that, if the approach speed $(V)$ of the bird is known or estimable, the width $w$ of the gap can be inferred from the relationship

$$
\frac{d \theta}{d t}=\left(\frac{4 V}{w}\right) \sin ^{2}\left(\frac{\theta}{2}\right)
$$

where $\theta$ is the angular width of the gap on the retinal image, and $\frac{d \theta}{d t}$ is the observed rate of change of this angular width.

Figure 8, based on Equation (1) shows the way in which $\frac{d \theta}{d t}$ is expected to vary with $\theta$, for a number of different gap widths $(w)$. In addition, the figure shows that if the speed of approach is known (it is assumed to be $4 \mathrm{~m} / \mathrm{s}$ in this example), the width of the gap can be estimated by monitoring the profile of the rate of expansion of the image of the gap as it is approached, or, alternatively, by sensing the rate of expansion of the image when it has a particular angular width. For example, when the angular width of the gap is 90 degrees, as shown by the dashed line, an expansion rate of 1240 degrees/second would indicate that the gap has a physical width of $36 \mathrm{~cm}$; an expansion rate of 1500 degrees/second would indicate a gap width of $30 \mathrm{~cm}$; and so on. If a bird with a wingspan of $34 \mathrm{~cm}$ experiences an image expansion of 1600 degrees/second, it would have to close its wings because the indicated gap width is $28 \mathrm{~cm}$.

In principle, the relationships shown in Figure 8 can be used to determine if closure is necessary and when to close the wings. Let us assume that each bird carries within its nervous system a representation of the characteristic function that corresponds to a gap of width equal to its wingspan. If the bird approaches a gap that is narrower than its wingspan, the rate of change of gap width will be consistently higher than that carried in the internal representation, at each visual angle. The "confidence" of the hypothesis (gap < wingspan) can therefore be built up by integrating the differences that are sensed

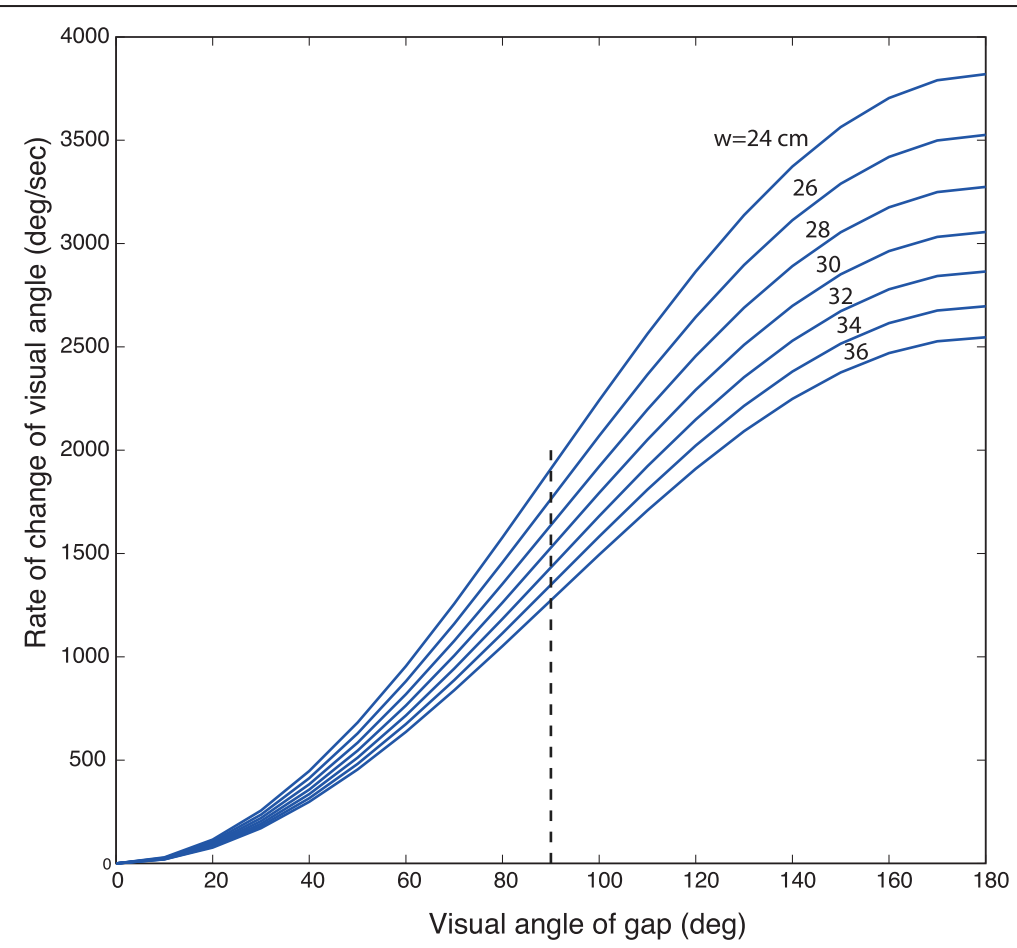

Figure 8 Determining whether, and, if so, when, to close the wings. The plot shows the expected rate of expansion of the image of a gap as it is approached, as a function of the instantaneous visual angle subtended by the gap, with the physical width of the gap as a parameter. The vertical dashed line represents an angular width of $90 \mathrm{deg}$. These curves are plotted assuming an approach speed of $4 \mathrm{~m} / \mathrm{s}$, based on analysis of video sequences (Vo, Schiffner and Srinivasan, unpublished data). 
at successive visual angles as the gap is approached. A decision to close the wings can be made when this integrated difference exceeds an internally set threshold. With such a scheme, wing closure will occur at a relatively small visual angle (i.e. at a relatively large distance from the gap) when the gap is much narrower than the bird's wingspan, at a larger visual angle (i.e. at a relatively small distance from the gap) when the gap is only marginally narrower than the wingspan, and never when the gap is wider than the wingspan.

This strategy for estimating gap width and triggering wing closure would require information about the speed of approach. It is not yet known whether birds are able to estimate the speed of their flight. In principle, flight speed can be estimated by sensing the air speed, or calibrating it in terms of the parameters of the wing stroke (such as the amplitude, frequency, plane and wing articulation). One way to test the visual expansion strategy would be to examine whether wing closure occurs at larger distances for narrower gaps, as predicted above. Another way would be to film the birds' behaviour when the width of the gap is varied dynamically during the approach.

\section{Conclusion}

In summary, our findings indicate that budgerigars are very precise at estimating the widths of passages and assessing their navigability. Birds are individually aware of their own wingspan, and are capable of combining this "body image" with visual information from the environment to orchestrate their flight through narrow passages.

\section{Materials and methods}

\section{Ethics statement}

All experiments were carried out in accordance with the Australian Law on the protection and welfare of laboratory animals and the approval of the Animal Experimentation Ethics Committees of the University of Queensland, Brisbane, Australia (QBI/303/12/ARC).

\section{Subjects}

The subjects were seven adult male wild type budgerigars (Melopsittacus undulatus) between three and five years old. They were purchased from a pet shop at an age of approximately one month and were housed in an outdoor aviary. The aviary was $4.0 \mathrm{~m}$ in length, $2.0 \mathrm{~m}$ in width and $2.2 \mathrm{~m}$ in height, and had large mesh-screened windows, which provided natural light and a diurnal light cycle. Perches were provided in areas that presented sunshine, as well as in areas that offered protection from inclement weather. The experiments were conducted at The University of Queensland's Pinjarra Hills field station. On the days in which experiments were conducted (usually two to three days per week), the birds were kept in groups of up to four in smaller cages of $47 \mathrm{~cm}$ length, $34.5 \mathrm{~cm}$ width and $82 \mathrm{~cm}$ height for a period not exceeding eight hours, to permit easy access to individual birds for experimentation. At the end of a day's experimentation the birds were moved back to the aviary.

\section{Training}

The birds were trained to take off at one end of a tunnel from a perch, which was either held by an experimenter or fixed on the wall, and to fly to the other end of the tunnel. To provide proper motivation for the birds, a cage with other familiar birds was placed at the other end of the tunnel. Further into the experiment this motivational stimulus proved to be unnecessary, but the procedure was continued in order to preserve uniformity across all of the experiments.

\section{Apparatus and implementation}

The experiments took place indoors in a tunnel that was $7.28 \mathrm{~m}$ long, $1.36 \mathrm{~m}$ wide and $2.44 \mathrm{~m}$ high, with white walls and ceiling, and a grey floor (see Figure 1). The end walls were covered with black cloth, to standardise visual cues. In the middle of the tunnel the birds were presented with an aperture of varying width, formed by two sheets of cloth suspended from the ceiling. The sheets carried a checkerboard pattern (check size $4 \mathrm{~cm}$ ) making the aperture and the flanking panels clearly visible to the birds.

The width of the gap was varied in a pseudo random fashion, in separate flight trials. This width ranged from 24 to $38 \mathrm{~cm}$, and was chosen to bracket the birds' wingspans, which were 29(1 bird), 31(3 birds) and 33( 3 birds) $\mathrm{cm}$ (measurements were rounded to the nearest $\mathrm{cm}$ ). Consequently, for all birds, the absolute differences between gap width and wingspan varied at least over the range -5 to $+5 \mathrm{~cm}$. In one additional trial, the gap had a width of $13 \mathrm{~cm}$, which was narrow enough that all of the birds would be forced to move both wings out of the way while traversing the gap.

\section{Recording}

The birds' flights were filmed using a high-speed camera (DRS data \& imaging systems, Inc., Oakland, NJ). Camera operation and video acquisition were controlled by special-purpose software (MiDAS 2.0 (Xcitex, Inc., Cambridge, MA)). The camera was mounted on the ceiling, $50 \mathrm{~cm}$ in front of the gap, with its optical axis pointing vertically downwards (Figure 1). The camera had a visual field of 110 degrees along the tunnel axis and 93 degrees in the transverse direction, which enabled flight trajectories to be filmed from about $2.0 \mathrm{~m}$ ahead of the gap to about $1.0 \mathrm{~m}$ beyond it, the exact figures depending up the height of the bird's flight. All flights were recorded at 
200 frames per second, which provided sufficient temporal resolution to investigate the wingbeat cycle before, during and after passage through the gap. From these recordings we determined the times when each upstroke and each downstroke had been completed.

\section{Statistics}

In order to check for significant differences in the birds' behaviour across the different gap widths, we used both one and two way ANOVA with repeated measurements with a factorial design to test for differences in probability of wing-closure and normalized duration of the upstroke. The independent factors were the size of the gap (one way ANOVA) and the wingspan of the bird (additionally for the two way ANOVA). Each bird was treated as a repeated measure within each wingspan-category. When the ANOVA indicated significance, we used the Tukey HSD test -correcting for multiple comparisons - to verify the findings of the ANOVA and later the Wilcoxon signed Rank test for paired data to determine the critical gap width at which the birds started to fold their wings. For each ANOVA we ensured beforehand that neither normality, homogeneity of variances, nor sphericity had been violated using, Shapiro-Wilks test for normality, Levene's Test for homogeneity of variance and Mauchly's test for sphericity respectively.

\section{Additional files}

Additional file 1: Examples of high-speed videography of the budgerigars' flights while traversing the gap. Video shows a bird 'projectiling' through the gap with its wings almost completely tucked back. Bird shown is Drongo (wingspan $31 \mathrm{~cm}$ ), the gap size in the example is $28 \mathrm{~cm}$.

Additional file 2: Examples of high-speed videography of the budgerigars' flights while traversing the gap. Video shows a budgerigar traversing the gap with its wings held above its body for a prolonged time. Bird shown is One (wingspan $31 \mathrm{~cm}$ ), the gap size is $28 \mathrm{~cm}$.

\section{Competing interests}

The authors declare that they have no competing interests.

\section{Authors' contributions}

IS participated in the design of the study, carried out the experiments, performed the statistical analysis, and drafted the manuscript. HDV helped to carry out the experiments and analysed the raw video footage. PSB participated in the design of the study, helped to carry out the experiments, and analysed the raw video footage. MVS participated in the design of the study, and helped to draft the manuscript. All authors read and approved the final manuscript.

\section{Acknowledgements}

This research was supported by ARC Discovery Grant DP0559306, ARC Centre of Excellence in Vision Science Grant CE0561903, HFSP Research Grant RGP0003/2013, and by a Queensland Smart State Premier's Fellowship. We thank Lena Heeman and Brianna Hey for their help in conducting the experiments.

\section{Author details}

'Queensland Brain Institute, University of Queensland, St Lucia QLD 4072, Australia. ${ }^{2}$ School of Information Technology and Electrical Engineering, University of Queensland, St Lucia QLD 4072, Australia. ${ }^{3}$ ARC Centre of Excellence in Vision Science, University of Queensland, St Lucia QLD 4072, Australia.

Received: 19 May 2014 Accepted: 27 August 2014

Published: 4 September 2014

\section{References}

1. Peregrine Falcon and Gos Hawk in flight. In [http://www.youtube.com/ watch? $=$ = - - RHRAzUHM]

2. Martin GR: Visual fields and their functions in birds. J Ornitho/ 2007, 148:547-562.

3. Martin GR: Understanding bird collisions with man-made objects: a sensory ecology approach. Ibis 2011, 153:239-254.

4. Tobalske BW: Morphology, velocity, and intermittent flight in birds. Am Zool 2001, 41:177-187.

5. Bhagavatula P: Visually guided flight in birds using the Budgerigar (Melopsittacus undulatus) as a model system. Ph. D. Thesis. Australian National University; 2011.

6. Warren WH Jr, Whang S: Visual guidance of walking through apertures: Body-scaled information for affordances. J Exp Psychol Human 1987, 13:371.

7. Wilmut K, Barnett AL: Locomotor behaviour of children while navigating through apertures. Exp Brain Res 2011, 210:185-194.

8. Fath AJ, Fajen BR: Static and dynamic visual information about the size and passability of an aperture. Perception 2011, 40:887.

9. Franchak JM, Celano EC, Adolph KE: Perception of passage through openings depends on the size of the body in motion. Exp Brain Res 2012, 223:1-10.

10. Ishak S, Adolph KE, Lin GC: Perceiving affordances for fitting through apertures. J Exp Psychol Human 2008, 34:1501.

11. Lock $A$, Collett T: The three-dimensional world of a toad. Proc $R$ Soc $B$ 1980, 206:481-487.

doi:10.1186/s12983-014-0064-y

Cite this article as: Schiffner et al:: Minding the gap: in-flight body awareness in birds. Frontiers in Zoology 2014 11:64.

\section{Submit your next manuscript to BioMed Central and take full advantage of:}

- Convenient online submission

- Thorough peer review

- No space constraints or color figure charges

- Immediate publication on acceptance

- Inclusion in PubMed, CAS, Scopus and Google Scholar

- Research which is freely available for redistribution 